\title{
TESTS DE RAICES UNITARIAS ESTACIONALES EN LA SERIE CRONOLOGICA DE LOS PRECIOS DEL NOVILLO EN EL MERCADO DE LINIERS (ARGENTINA)
}

Antonio Aguirre*

\section{Introducción}

El estudio de las propiedades estacionales de las series cronológicas económicas ha sido uno de los temas de investigación que más atención recibió de los econometristas en los últimos años [ver, inter alia, Beaulieu y Miron (1992, 1993), Ghysels (1994), Harvey y Scott (1994), Hylleberg (1992), Hylleberg et al. (1993), Miron (1994), Osborn (1990)]. La evidencia proporcionada por estos estudios indica que, además de ser no estacionarias en la frecuencia cero, muchas series cronológicas económicas observadas con periodicidad inferior a la anual muestran también variaciones estacionales que son más grandes y más irregulares que lo que se creía anteriormente. De hecho, mientras algunas variables muestran variaciones estacionales determinísticas, otras despliegan movimientos estacionales que cambian lentamente a través del tiempo (un modelo mixto también parece ser común). En otras palabras, algunas series cronológicas económicas observadas trimestral o mensualmente parecen desplegar a menudo variaciones estacionales estocásticas no estacionarias y, en esos casos, los correspondientes procesos generadores de los datos (PGDs) son llamados estacionalmente integrados ${ }^{1}$ o procesos con raíces unitarias estacionales.

Cuando trabaja con datos que presentan estacionalidad, el investigador

\footnotetext{
*Profesor de la Facultad de Ciencias Económicas de la Universidad Federal de Minas Gerais e investigador del CEPE (Centro de Pesquisa em Economia Internacional).

'El concepto de integración estacional puede significar cosas diferentes para distintos autores. En la próxima sección serán discutidas algunas definiciones alternativas.
} 
puede utilizar algún filtro para obtener datos estacionalmente ajustados (un procedimiento como ese fue seguido por Hache (1974) estudiando la demanda de dinero) o puede intentar captar la estacionalidad por medio de variables 'dummy' estacionales lo que es equivalente a suponer que las variaciones estacionales son puramente deterministicas. "Sin embargo, si los efectos estacionales cambian gradualmente a través del tiempo, este (segundo) procedimiento produce una especificación dinámica errada" (Harvey y Scott, 1994, página 1324). Por esta razón, siempre que se usen datos estacionales en econometría parece aconsejable hacer el test de las propiedades estacionales de las series cronológicas utilizadas, ya que ese procedimiento es preferible a suponer la adecuación de cualquier especificación del modelo.

En la actualidad, el número de métodos disponibles para ayudar a distinguir entre movimientos estocásticos estacionarios y no estacionarios (posiblemente alrededor de componentes determinísticas) en las series cronológicas económicas con periodicidad menor que un año, es bastante grande. Esto transforma la elección de una estrategia de test en una tarea delicada donde el conocimiento de los 'pros' y 'contras' de cada procedimiento es de capital importancia. Este artículo intenta hacer una contribución discutiendo la aplicación de diferentes procedimientos y técnicas para determinar las propiedades estacionales de datos trimestrales en el caso particular de la serie de precios del novillo en el Mercado de Liniers (Argentina).

El trabajo está organizado como sigue. En la Sección 2 se presentan las clases usuales de procesos estacionales de series cronológicas y tres definiciones del concepto de integración estacional —indicando cuál de ellos será usado en este trabajo. La Sección 3 proporciona una breve sinopsis de dos tests de raíces unitarias estacionales que serán utilizados. Ellos son el de Hylleberg et al. (1990) [de aquí en adelante, HEGY] que es el procedimiento más ampliamente empleado para hacer el test de presencia de raices unitarias estacionales en la literatura aplicada actual, y los estadísticos propuestos por Canova y Hansen (1995) [de ahora en adelante, $\mathrm{CH}$ ] quienes cambian la hipótesis nula usual de que la serie no es estacionaria por la opuesta de que lo es. La descripción de los datos y los resultados de los diferentes tests se muestran en la Sección 4. La Sección 5 contiene las conclusiones del artículo. 
2. Procesos estacionales e integración estacional

La teoría subyacente al análisis de series cronológicas estacionales considera tres clases de procesos: aquellos puramente determinísticos, los estacionarios en covarianza, y los procesos estacionales integrados. La primera clase incluye aquellos procesos generados por componentes puramente determinísticos como un término constante, variables 'dummy' estacionales y tendencias determinísticas. En el siguiente ejemplo, el proceso $y_{1}$ - observado $s$ veces por año- es generado solamente por 'dummies' estacionales que afectan la coordenada al origen:

$$
y_{t}=\sum_{i=1}^{s} \alpha_{i} D i_{t}+\varepsilon_{1}
$$

donde las variables $D i,(i=1,2, \ldots, s)$ toman el valor 1 cuando $t$ corresponde a la estación $i$, y cero en los otros casos, y donde $\varepsilon_{1}$ es un conjunto de variables aleatorias idéntica e independientemente distribuídas (IID). Esta ecuación puede ser reformulada para evitar la confusión entre los niveles de las diferentes estaciones, de la siguiente manera:

$$
y_{1}=\mu+\sum_{i=1}^{s-1} \alpha_{i}^{*} D i_{i}^{*}+\varepsilon_{t}
$$

donde $\mu$ es la media del proceso y los coeficientes $\alpha_{i}{ }^{*}$ están sujetos a la restricción de sumar cero. Para hacer operativa esta restricción las 'dummies' $D i_{\mathrm{t}}{ }^{*} \quad(\mathrm{i}=1,2, \ldots, \mathrm{s})$ se definen de manera tal que toman valor 1 cuando $t$ corresponde a la estación $i,-1$ cuando $t$ representa la estación $s$, y valen cero el resto de las veces, y donde $\varepsilon_{t}$ es una serie de variables aleatórias IID.

Finalmente, la ecuación anterior también puede incluir tendencias determinísticas con coeficientes estacionales constantes o variables, es decir,

$$
y_{1}=\mu \quad+\sum_{i=1}^{s-1} \alpha_{i} D i_{t}+\sum_{i=1}^{s} \beta_{i}\left[D i_{l} \times g(t)\right]+\varepsilon_{t}
$$

donde $g(t)$ es un polinomio determinístico en $t$.

El segundo caso - proceso estacional estacionario en covarianzapuede ser ejemplificado por un modelo expresado como 


\section{ESTUDIOS ECONOMICOS}

$$
y_{t}=\rho_{s} y_{t}+\varepsilon_{\mathrm{t}}
$$

donde $\left|\rho_{s}\right|<1$ y $\varepsilon_{t}$ es un conjunto de variables aleatorias IID.

Si $p_{s}=1$ en la ecuación (4), entonces tenemos un paseo aleatorio estacional, un proceso que exhibe un patrón estacional que varía a través del tiempo. Ésta es la tercera clase de proceso estacional listada anteriormente. En ese caso, la variable $\Delta_{s} y_{1}$, definida como,

$$
\Delta_{\mathrm{s}} y_{\mathrm{t}}=y_{\mathrm{t}}-y_{\mathrm{t}-\mathrm{s}}
$$

es estacionaria. La diferencia principal entre estas formas de estacionalidad es que en el modelo determinístico, si $\varepsilon_{\mathrm{t}}$ es ruido blanco, los 'shocks' tienen sólo un impacto inmediato; en el caso del modelo estacional estacionario los 'shocks' tienen un efecto transitorio (desaparecen en el largo plazo), mientras que tienen un efecto permanente en el modelo integrado. Es decir, los procesos estacionalmente integrados tienen propiedades similares a aquéllas observadas en el caso ordinario (frecuencia cero). Las series «...tienen 'buena memoria' de manera tal que los efectos de un shock permanecen para siempre y pueden, en consecuencia, modificar el patrón estacional de manera permanente. Estos procesos tienen varianzas que aumentan linealmente desde el comienzo de la serie y no están asintóticamente correlacionados con procesos que tengan raíces unitarias en otras frecuencias» (Hylleberg et al., 1990, pág. 218).

Ilmakunnas demuestra que «la secuencia de los tests depende de la definición de integración estacional adoptada» (Ilmakunnas, 1990, página 97). De entre las diferentes definiciones de integración estacional existentes mencionamos tres de ellas: la propuesta por Osborn et al. (1988), otra debida a Engle et al. (1989) y finalmente aquélla dada por Hylleberg et al. (1990). Ilmakunnas reproduce las dos primeras definiciones: se dice que una variable es integrada de órdenes (d,D), denotado $I(d, D)$, si la serie se transforma en estacionaria después de diferenciarla $d$ veces con diferencias finitas y $D$ veces con diferencias estacionales, es decir, $X_{t} \sim \mathrm{I}(\mathrm{d}, \mathrm{D}) \mathrm{si}$ $(1-L)^{d}\left(1-L^{s}\right)^{D} X_{1}=\Delta^{d} \Delta_{s}^{D} X_{T}$ es estacionaria. El segundo concepto establece que una serie cronológica es integrada de orden $d_{0}$ y $d_{s}$ denotado $\operatorname{SI}\left(\mathrm{d}_{0}, \mathrm{~d}_{\mathrm{s}}\right)$ si $(1-L)^{d o}[S(L)]^{d s} X_{1}=\Delta^{d o}[S(L)]^{d s} X_{1}$, es estacionaria, donde la expresión polinómica $\mathrm{S}(\mathrm{L})$ se define como $S(L)=1+L+L^{2}+\ldots+L^{S-1}$ 
Cuando las variables no presentan integración estacional ambas definiciones coinciden, es decir, $\mathrm{I}(1,0)=\mathrm{SI}(1,0), \mathrm{I}(2,0)=\mathrm{SI}(2,0)$, etc. Por el contrario, siempre que una serie es estacionalmente integrada estas definiciones difieren. Esto sucede porque $\Delta_{s}=\left(1-L^{s}\right)$ puede factorearse como $(1-L) S(L)$. De esta manera, el equivalente de $\mathrm{I}(0,1)$ es $\operatorname{SI}(1,1)$; $\mathrm{I}(1,1)=\mathrm{SI}(2,1)$, y así sucesivamente. De la misma manera, el proceso $\mathrm{SI}(0,1)$ - usando la definición de Engle - no tiene equivalente si se usa el concepto de Osborn. En este trabajo utilizaremos la definición SI .

Finalmente, una tercera definición afirma que "una serie $x_{\text {, }}$ es un proceso estacional integrado si tiene una raíz unitaria estacional en su representación autoregresiva. Más generalmente, es integrada de orden $d$ en la frecuencia $\theta$ si el espectro de $\boldsymbol{x}_{\boldsymbol{t}}$ toma la forma

$$
f(\omega)=c(\omega-\theta)^{-2 d}
$$

para $\omega$ próxima a $\theta$. Esto es denotado convenientemente por $x_{t} \sim \operatorname{I} \theta(\mathrm{d})$ " (Hylleberg et al. 1990, pág. 217). Esta definición resulta conveniente al discutir los resultados de algunos tests, como se mostrará en la Sección 4.

\section{Tests de raices unitarias estacionales}

Antes de describir los diferentes tests que se usarán en este trabajo mencionaremos la estrategia propuesta por Dickey y Pantula (1987): estos autores sugieren que, para conservar el tamaño nominal del test en el caso de más de una raíz unitaria (en la frecuencia cero), es conveniente comenzar la sucesión de tests a partir del número máximo de raíces en consideración. Ilmakunnas (1990) conjetura que esto también es válido cuando se trabaja con datos trimestrales y presenta una sucesión completa de posibles tests que empiezan con el caso SI $(2,1)$, indicando las alternativas que pueden someterse a test en cada caso. Siguiendo este criterio en el caso que nos ocupa, comenzamos haciendo el test de la hipótesis de existencia de dos raíces unitarias en la frecuencia cero. Sin embargo, los resultados detallados de este test - que rechaza la nula- no son presentados, mostrándose unicamente los correspondientes al test de una única raíz unitaria en la mencionada frecuencia. 
Si una variable tiene que ser filtrada de alguna manera para que se transforme en estacionaria, esto puede ser causado por una raíz unitaria en la frecuencia cero [correspondiente a (1-L)], o por raíces unitarias de alguna frecuencia estacional [correspondientes a la descomposición de S(L)]. Este hecho determina cual será el modelo de regresión que debe ser estimado para hacer el test de una dada hipótesis nula. "La idea básica es que cuando la hipótesis nula establece que existe una raíz unitaria en el 'lag' de orden 1 o en los 'lags' estacionales, los estadísticos del test son modificados de manera tal que, al estimarse la regresión del test, se usen datos apropiadamente diferenciados (con $\Delta \circ \Delta_{s}$, respectivamente). Cuando la hipótesis mantenida es que existen raíces unitarias en las frecuencias estacionales, deben usarse datos estacionalmente corregidos [con el operador $S(L)$ ]) (Ilmakunnas, 1990, página 80).

\subsection{Tests con hipótesis nula de existencia de raíces unitarias}

El test de HEGY es una extensión del de Dickey-Fuller (Dickey y Fuller, 1979, 1981) para el caso en que la periodicidad de los datos es trimestral. Éste es el test más comunmente usado en los trabajos empíricos recientes. La estrategia propuesta por estos autores es más general que la de los procedimientos anteriores - para el mismo problema - ya que permite detectar la presencia de raíces unitarias en una (o en todas) las frecuencias estacionales así como en la frecuencia cero.

Para hacer el test de la hipótesis nula de que las raíces del polinomio autoregresivo $1-L^{4}=0$ están localizadas sobre el círculo unitario, versus la alternativa de que se encuentran fuera del mencionado círculo, los autores usan el factoreo de ese polinomio basado en las cuatro raíces del mismo

$$
\left(1-L^{4}\right)=(1-L)(1+L)(1-i L)(1+i L)
$$

donde cada factor del lado derecho de la igualdad está asociado con una raíz en particular. Agrupando los factores del lado derecho de (6) de tres maneras

$$
\begin{aligned}
(1-L)(1+L)(1-i L)(1+i L) & =(1-L) S(L) \\
& =(1+L)\left(1-L+L^{2}-L^{3}\right) \\
& =\left(1-L^{2}\right)\left(1-L^{2}\right)
\end{aligned}
$$


El segundo factor en el lado derecho de las tres igualdades anteriores, o sea $S(L),\left(1-L+L^{2}-L^{3}\right)$ y $\left(1-L^{2}\right)$, son filtros que - como será explicado a continuación - transforman adecuadamente los datos originales generando tres variables auxiliares utilizadas en la regresión del test. Cuando la hipótesis nula del test establece que existen dos raices unitarias en la frecuencia cero esos filtros se aplican sobre la primera diferencia de la variable en vez de aplicarlos sobre el nivel de la misma. Este último caso, que corresponde al test de una raíz unitaria, es el que usamos a continuación para mostrar el procedimiento de HEGY. Si denominamos $y$, a los precios e $Y i,(i=1,2,3)$ a las variables auxiliares, tenemos:

- $Y 1,=S(L) y$, la transformación de los datos que filtra toda la variación estacional de la serie reteniendo la raíz unitaria asociada a la raíz +1 dé polinomio autoregresivo (frecuencia cero);

$-\mathrm{Y}_{1}=-\left(1-\mathrm{L}+\mathrm{L}^{2}-\mathrm{L}^{3}\right)$ es la transformación que retiene la raíz unitaria -1 del polinomio autoregresivo, la cual está asociada con la frecuencia $\pi .^{2} \mathrm{El}$ cambio de signo del filtro tiene por objeto facilitar la manipulación algebraica mas no afecta la esencia del test;

- $\mathrm{Y}_{\mathrm{t}}=-\left(1-\mathrm{L}^{2}\right) y_{\mathrm{t}}$ es la variable auxiliar asociada con las dos raíces complejas conjugadas del polinomio autoregresivo y que corresponde a la frecuencia $\frac{\pi}{2}$.

Cabe resaltar, por lo tanto, que se cumplen las siguientes igualdades:

$$
\begin{aligned}
& \Delta_{4} y_{1}=(1-L)\left(1+L+L^{2}+L^{3}\right) y_{1}=(1-L) Y 1 \\
& \Delta_{4} y_{1}=(1+L)\left(1-L+L^{2}-L^{3}\right) y_{1}=-(1+L) Y 2, \\
& \Delta_{4} y_{1}=\left(1+L^{2}\right)\left(1-L^{2}\right) y_{1}=-\left(1+L^{2}\right) Y 3
\end{aligned}
$$

Después de hacer uso de algunos resultados del álgebra, los autores obtienen una expresión equivalente que facilita el test de hipótesis. El modelo testable resultante, usado para verificar la presencia de una raiz unitaria ${ }^{3}$ en

2 Los detalles relacionados con las raices unitarias del polinomio autoregresivo y las correspondientes frecuencias están explicados de forma intuitiva en el Apéndice 1.

${ }^{3}$ En este caso la hipótesis nula de existencia de dos raíces unitarias en la frecuencia cero ya fue rechazada. El modelo usado en aquel test es semejante a la ecuación (8): la variable dependiente es $\Delta \Delta_{12} y_{1}$ y las auxiliares fueron obtenidas con los filtros anteriores, apenas que aplicados a $\Delta y_{1}$ en vez de $y_{1}$. 
la frecuencia cero y raíces unitarias en las frecuencias estacionales, está dado por la ecuación (8) que puede ser estimada por mínimos cuadrados ordinarios (MCO) y los estadísticos $\pi$ usados para realizar inferencias, mientras que el término $\mu_{t}$ puede contener una constante, una tendencia deterministica y/o tres 'dummies' estacionales:

$$
\begin{aligned}
\Delta_{4} y_{1} & =\mu_{t}+\pi_{1} Y 1_{i-1}+\pi_{2} Y 2_{t-1}+\pi_{4} Y 3_{t-1}+ \\
& +\pi_{3} Y 3_{t-2}+\sum_{i=1}^{k} \delta_{i} \Delta_{4} y_{t-i}+\varepsilon_{t}
\end{aligned}
$$

El orden de los 'lags' (el valor de $k$ en la suma) es determinado usando tests de diagnóstico de manera tal que los errores del modelo estimado sean ruido blanco. En la próxima sección se informa sobre los resultados del ajuste de esta ecuación. La interpretación de los resultados y los valores críticos necesarios para realizar los tests pueden encontrarse en Hylleberg et al. (1990). En la estimación de la ecuación (8), si no se rechaza la nula de $\pi_{1}=0$ significa que existe una raíz unitaria en los datos estacionalmente ajustados. No rechazar $\pi_{2}$ implica en la existencia de una raíz unitaria en el ciclo estacional con periodo semianual (dos ciclos por año). Si $\pi_{3}$ y $\pi_{4}$ son estadísticamente iguales a cero existe una raíz unitaria en el ciclo estacional anual.

\subsection{Tests con hipótesis nula de estacionariedad}

Puesto que los tests de HEGY toman como nula la existencia de raíces unitarias, "el rechazo de su hipótesis nula implica el resultado (fuerte) que la serie tiene un comportamiento estacional estacionario. Sin embargo, debido a la baja potencia de los tests en muestras de tamaño moderado, la falta de rechazo de la hipótesis nula no puede tomarse como evidencia contundente 'a favor' de la presencia de raíces unitarias" (Canova y Hansen, 1995, página 237). Teniendo en cuenta este problema de baja potencia del test HEGY, un complemento útil de los procedimientos anteriores sería otro test que tomase la estacionalidad estacionaria como hipótesis nula y la no estacionaria como alternativa. "En este cpntexto, el rechazo de la hipótesis 
nula implicaría el resultado (fuerte) de que los datos son de hecho no estacionarios, una conclusión que ni los tests DHF ni los de HEGY pueden proporcionar. Visto juntamente con estos tests, semejante procedimiento permitiría a los investigadores realizar un análisis más completo de sus datos" (Canova y Hansen, 1995, página 238).

El punto de partida para estos autores es un modelo lineal de series cronológicas con estacionalidad estacionaria que puede especificarse matemáticamente de dos maneras diferentes, aunque equivalentes. La primera es la representación trigonométrica usada en la literatura de serie cronológicas, ${ }^{4}$ y la segunda es la formulación con variables 'dummy'. El primer procedimiento da lugar a dos tests de raíces unitarias en las frecuencias estacionales y el segundo resulta en cuatro tests para la variación en el tiempo de los coeficientes de las variables 'dummy' estacionales (datos trimestrales). Estos diferentes tests son obtenidos mediante la especificación apropiada de la hipótesis alternativa en cada caso. La regresión auxiliar usada para realizar estos tests es la siguiente:

$$
y=\mu+Z^{\prime \prime} \beta+f_{1}^{\prime} \gamma+u_{t}
$$

donde $Z_{1}$, es un vector $(\mathrm{k} \times 1)$ de variables explicativas, $u_{t}$ es estacionario $f_{1}^{\prime}=\left[\cos \left(\theta_{1}, t\right), \sin \left(\theta_{1}, t\right), \ldots, \cos \left(\theta_{s / 2}, \mathrm{t}\right), \sin \left(\theta_{s} / 2, \mathrm{t}\right)\right]$, donde $\theta_{j}=\frac{2 \pi j}{s}(j=1,2, \ldots s / 2)$ y $s$ es el número de observaciones por año. De esta manera, $f_{t}$ es equivalente a un conjunto de variables 'dummy' estacionales representado en el dominio de la frecuencia.

Si la alternativa en consideración es 'estacionalidad no estacionaria', entonces debe hacerse un test simultáneo de la existencia de raíces unitarias en todas las frecuencias estacionales. Esto significa que, para ejecutar el test de estabilidad con hipótesis nula de estacionariedad en todas las frecuencias, el estadístico que debe calcularse es el siguiente:

${ }^{4}$ Ver Priestley (1981) y Aguirre (1995). 


$$
\begin{gathered}
L_{f}=T^{-2} \sum_{t=1}^{T} \widetilde{F}_{t}^{\prime}\left(\widetilde{\Omega}^{f}\right)^{-1} \widetilde{F}_{t} \\
=T^{-2} \operatorname{tra}\left[\left(\widetilde{\Omega}^{f}\right)^{-1} \sum_{t=1}^{T} \widetilde{F}_{t} \widetilde{F}_{t}\right] \\
\text { donde } \widetilde{F}_{t}=\sum_{t=1}^{T} f_{l} \widetilde{u}_{t} \text { es una secuencia de sumas parciales, } \widetilde{u}_{t} \text { es }
\end{gathered}
$$

el conjunto de residuos en la estimación de la ecuación (9) por MCO y donde

$$
\widetilde{\Omega}^{f}=\sum_{k=-m}^{m} W(k, m) \frac{1}{T} \sum_{l} f_{1+k} f_{1}^{\prime} \tilde{u}_{t+k} \tilde{u}_{t}
$$

es una estimación consistente de la matriz de varianza-covarianza de $f, \widetilde{u}_{\text {, }}$ (llevando en cuenta la posible existencia de heterocedasticidad y autocorrelación), y donde W(.,.) es una ventana de alisado ('smoothing').

Si lo que interesa es hacer el test de los componentes estacionales en las correspondientes frecuencias individuales, la matriz pertinente asume una forma diferente y el estadístico $L$ original se reduce a $L \theta_{j} \quad(j=1,2, \ldots$, $s / 2$ ) que puede computarse como un subproducto del cálculo de $L_{f}$. Cuando se usan datos trimestrales $(s=4)$ resultan dos estadísticos de ese tipo. Éstos son dados por la forma cuadrática

$$
\begin{aligned}
& L_{\theta, j}=T^{-2} \sum_{t=1}^{T} \widetilde{F}_{j i}^{\prime}\left(\widetilde{\Omega}_{j i}^{f}\right)^{-1} \widetilde{F}_{j t} \\
& \widetilde{F}_{j t}=\sum_{i=1}^{T} f_{j t} \widetilde{u}_{t}, f_{j t}^{\prime}=\left[\cos \left(\theta_{j}, t\right), \sin \left(\theta_{j}, t\right)\right], \quad f_{s / 2, t}=\cos (\pi, t)=(-1)^{\prime} \quad \mathrm{y}
\end{aligned}
$$

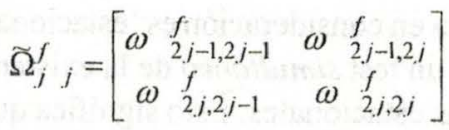

$$
\begin{aligned}
& \text { para } j<\frac{s}{2} \text { y donde } \omega_{h l}^{f} \text { es un elemento característico de } \widetilde{\Omega}_{j, j}^{f} \text {. }
\end{aligned}
$$


La distribución asintótica del estadístico del test es la distribución generalizada de Von Misses con grados de libertad según la dimensión del proceso de sumas parciales. "Los tests $L \theta_{j}$ son complementos útiles del test conjunto $L_{f}$. $\mathrm{Si}$ el test conjunto rechaza la nula esto podría deberse a la existencia de raíces unitarias en cualquiera de las frecuencias estacionales. Los tests $L \theta_{j}$ son aptos para descubrir exactamente en cual frecuencia estacional específica surge la no estacionariedad» (Canova y Hansen, 1995, página 242). Para realizar el test de patrones estacionales variables se usa el modelo más tradicional con variables 'dummy' estacionales, determinando si la coordenada al origen estacional cambia a través del tiempo. Nuevamente, escogiendo apropiadamente la forma de la matriz pertinente es posible definir $s$ estadísticos $L_{a}$ diferentes $(a=1, \ldots, s)$ que permiten hacer el test de la estabilidad de la $a$ ésima ordenada al origen estacional. Cuando el objetivo del test es la estabilidad conjunta de las constantes estacionales se define un estadístico $L_{r}$. Sin embargo, éste es un test para inestabilidad en cualquier ordenada al origen estacional, de manera que pueden detectarse hasta movimientos de largo plazo (frecuencia cero). Como resultado, la hipótesis nula puede rechazarse en consecuencia de la existencia de inestabilidad de largo plazo en esa frecuencia, lo cual es un rasgo indeseable del test. Las modificaciones propuestas por Canova y Hansen para resolver este problema los llevaron de vuelta al estadístico del test conjunto definido en el primer caso. Este resultado llevó a los autores a hacer el siguiente comentario: «Para decirlo con otras palabras, hemos descubierto que ambas construcciones, testando la inestabilidad del punto de vista de las constantes estacionales o mirando del ángulo de las raíces unitarias estacionales, resulta en exactamente el mismo test conjunto» (Canova y Hansen, 1995, página 243).

4. Descripción de los datos y resultados de los tests

Los datos para este trabajo tienen, básicamente, dos fuentes. La primera es la tesis de doctorado de Gustavo Nores (Nores, 1972) donde encontramos el precio medio de exportación, por trimestre, de la tonelada de carne bovina argentina. Esos datos, que cubren el periodo 1960-1970, están 
expresados en pesos de 1960. Según el autor, la principal fuente de esos datos fue la Junta Nacional de Carnes.

La segunda fuente es una publicación de la Dirección de Mercados Ganaderos de la Secretaría de Agricultura, Ganadería y Pesca (SAGyP, 1994) donde se publica la serie mensual del índice del precio real del $\mathrm{kg}$ vivo del novillo en el Mercado de Liniers. Esta serie cubre el periodo enero de 1970octubre de 1994.

Los productos a los cuales corresponden los precios de las dos series arriba mencionadas son diferentes. Por ese motivo, el valor medio de la serie de Nores es de $25.820,00$ pesos $\mathrm{m} / \mathrm{n}$ de 1960 por tonelada, mientras que la media de la serie de la SAGyP es de $18.420,00$ pesos $\mathrm{m} / \mathrm{n}$ de 1960 por tonelada. Cabe mencionar que, como se comentará más adelante, al final de 1974 hubo un cambio estructural significativo en la serie del precio de la carne lo que puede explicar, por lo menos en parte, el nivel medio menor de la segunda serie.

Con la intención de disponer de una serie más larga, se calcularon las medias simples, por trimestre, de la serie de la SAGyP. Expresando ambas series trimestrales como índices con base de comparación localizada en el primer trimestre de 1970 , la primera serie tiene media aritmética de 119,92 y la segunda de 98,48 .

La diferencia de las dos series se pone en evidencia al examinar los cuatro valores de los respectivos índices correspondientes al único periodo común, o sea, el año de 1970. Con efecto, mientras que la serie de Nores aumenta en la progresión $100,109,11,118,5$ y 132,10, la segunda lo hace en la forma $100,109,06,121,14$ y 143,96. La tendencia es la misma, pero el ritmo de crescimiento es mayor en el caso de la serie de la SAGyP. De cualquier manera, el encadenamiento de las dos series es casi perfecto, como puede observarse en el Gráfico I. 
Gráfico I: Precio trimestral del novilho enel mercado de Liniers (Indice de los precios reales; base de comparación: 1970-I)

Período: 1960-1994

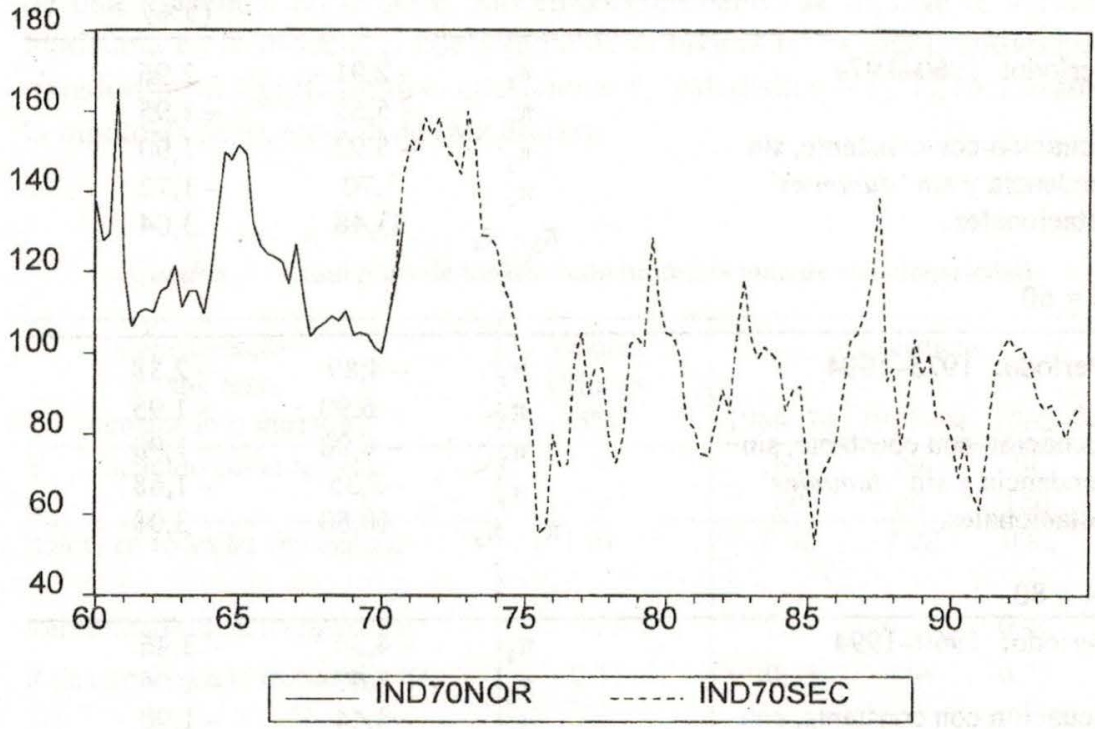

La observación visual del gráfico anterior revela una acentuada dismininución del índice entre 1973 y 1975. Con efecto, el índice cae de un valor de 160 en el primer trimestre de 1973 para 55 en el tercer trimestre de 1975. Cabe resaltar que esa disminuición ocurre en la serie de la SAGyP, lo cual elimina la posibilidad de que el problema se origine en el encadenamiento de las series. En consecuencia, es evidente que los datos correspondientes al periodo 1960-1974 tienen un nivel medio superior al resto de la serie.

Usando el test de Chow (1960) en un modelo de regresión con una 'dummy' para la ordenada al origen y otra para la pendiente, se detecta una ruptura estructural en la serie al final de 1974. Por lo tanto, el último trimestre de 1974 y el primero de 1975 pertenecen a dos 'conjuntos de datos' diferentes. La primera mitad tiene 60 observaciones y valor medio de 125,56 mientras que la segunda, con 80 observaciones, tiene media igual a 89,96.

Oportunamente comentaremos sobre los efectos que esta ruptura estructural tiene sobre los tests que realizamos en este trabajo. Los mismos serán efectuados para los dos subconjuntos separadamente y para la muestra completa con la finalidad de observar si tal procedimiento modifica nuestras conclusiones sobre las propiedades estadísticas de la serie analizada. 
Cuadro I: Resultados de los tests con hipótesis nula de existencia de raíces unitarias

\begin{tabular}{|c|c|c|c|}
\hline TEST & Parámetro & Estadístico & $\begin{array}{c}\text { Valor crítico } \\
\qquad(5 \%)\end{array}$ \\
\hline $\begin{array}{l}\text { Período: } 1960-1974 \\
\text { Ecuación con constante, sin } \\
\text { tendencia y sin 'dummies' } \\
\text { estacionales. } \\
\mathrm{N}=60\end{array}$ & $\begin{array}{c}\pi_{1} \\
\pi_{2} \\
\pi_{3} \\
\pi_{4} \\
\pi_{3 \cap} \pi_{4}\end{array}$ & $\begin{array}{c}-2,91 \\
-5,63 \\
-5,92 \\
-3,70 \\
33,48\end{array}$ & $\begin{array}{r}-2,96 \\
-1,95 \\
-1,90 \\
-1,72 \\
3,04\end{array}$ \\
\hline $\begin{array}{l}\text { Período: } 1975-1994 \\
\text { Ecuación con constante, sin } \\
\text { tendencia y sin 'dummies' } \\
\text { estacionales. } \\
\mathrm{N}=80\end{array}$ & $\begin{array}{c}\pi_{1} \\
\pi_{2} \\
\pi_{3} \\
\pi_{4} \\
\pi_{3 \curvearrowleft} \pi_{4}\end{array}$ & $\begin{array}{c}-4,89 \\
-6,90 \\
-6,90 \\
-3,35 \\
40,80\end{array}$ & $\begin{array}{r}-2,88 \\
-1,95 \\
-1,90 \\
-1,68 \\
3,08\end{array}$ \\
\hline $\begin{array}{l}\text { Período: } 1960-1994 \\
\text { Ecuación con constante, con } \\
\text { tendencia y sin 'dummies' } \\
\text { estacionales. } \\
N=140\end{array}$ & $\begin{array}{c}\pi_{1} \\
\pi_{2} \\
\pi_{3} \\
\pi_{4} \\
\pi_{3} \curvearrowleft \pi_{4}\end{array}$ & $\begin{array}{r}-4,34 \\
-8,67 \\
-8,44 \\
-5,20 \\
68,25\end{array}$ & $\begin{array}{r}-3,46 \\
-1,96 \\
-1,90 \\
-1,64 \\
3,04\end{array}$ \\
\hline
\end{tabular}

En el Cuadro I presentamos los estadísticos estimados y los valores críticos necesarios para realizar los varios tests de HEGY. En los tres periodos fue ajustado el modelo dado por la ecuación (8), con las diferencias indicadas en el cuadro, es decir, en todos los casos se incluye una constante mientras que solamente en el periodo completo se incluye una tendencia determinística. Como los coeficientes de las variables 'dummy' estacionales no fueron significativos tales variables fueron eliminadas de los modelos. En ninguno de los tres casos fue necesario incluir la variable dependiente retrasada para obtener residuos sin autocorrelación.

Exceptuando el caso del coeficiente $\pi_{1}$ en el periodo 1960-1074, en todos los otros casos se rechaza la hipótesis nula de existencia de raíces unitarias al $5 \%$ de significación. Antes de concluir que existe una raiz unitaria en la frecuencia cero de la serie del primer periodo, recordemos las 
advertencias de Canova y Hansen (1995) sobre la baja potencia de ios tests de HEGY. Si esa raíz unitaria realmente existiera eso implicaría la presencia de una tendencia en la serie. Sin embargo, cuando se incluye la variable tendencia en el modelo el coeficiente de la misma no es significativo pero transforma en significativo el coeficiente $\pi_{1}$ (estadístico $-3,21$ ), rechazando la hipótesis de existencia de raíz unitaria.

Cuadro II: Resultados de los tests con hipótesis nula de estacionariedad

\begin{tabular}{|c|c|c|c|c|}
\hline $\begin{array}{c}\text { Características } \\
\text { de los tests } \\
\text { (tamaño de la muestra) }\end{array}$ & $\begin{array}{l}\text { Valores } \\
\text { Críticos } \\
(5 \%)\end{array}$ & $1960-74$ & Estadístico & 1960-94 \\
\hline $\mathrm{N}^{1-1}$, Incluido como regresor & - & $\begin{array}{l}\text { No } \\
60 \\
\end{array}$ & $\begin{array}{l}\text { No } \\
80 \\
\end{array}$ & $\begin{array}{c}\text { Sí } \\
140 \\
\end{array}$ \\
\hline Raíces en todas las frecuencias & 1,01 & 0,46 & 0.28 & 0,82 \\
\hline Raíz unitaria en la frecuencia $\pi$ & 0,75 & 0,37 & 0,24 & 0,49 \\
\hline $\begin{array}{l}\text { Raíz unitaria en la frecuencia } \pi / 2 \\
\text { Inestabilidad por trimestre: }\end{array}$ & 0,47 & 0,13 & 0,04 & 0,35 \\
\hline Trimestre 1 & 0,47 & 0,20 & 0,04 & 0,28 \\
\hline Trimestre 2 & 0,47 & 0,13 & 0,13 & 0,15 \\
\hline Trimestre 3 & 0,47 & 0,10 & 0,10 & 0,44 \\
\hline Trimestre 4 & 0,47 & 0,28 & 0,12 & 0,23 \\
\hline Inestabilidad en todos los trimestres & 1,24 & 0,71 & 0,38 & 1,23 \\
\hline
\end{tabular}

Estos resultados son semejantes a los obtenidos en el análisis de la serie de precios del novillo en el Estado de São Paulo, Brasil (Aguirre, 1997). En ese caso los tests de HEGY tampoco detectaron raíces unitarias estacionales, pero no consiguieron rechazar la hipótesis nula de existencia de una raíz unitaria en la frecuencia cero. Además, la serie de precios brasileña presenta estacionalidad determinística significativa, característica que no está presente en la serie de precios del mercado de Liniers.

Acerca de los resultados obtenidos con la aplicación de la metodología de $\mathrm{CH}$ (ver Cuadro II), los tests fueron ejecutados con la serie de los precios 
sin ninguna transformación. En los dos subperiodos no se incluyó la variable dependiente retrasada entre los regresores, pero sí se lo hizo en el caso de la serie completa debido a la presencia de la tendencia decreciente ya comentada. Los datos del Cuadro II informan los valores de los estadísticos necesarios para realizar el test conjunto de existencia de raíces unitarias en todas las frecuencias y en cada una de las frecuencias estacionales. También se presentan aquellos estadísticos con los cuales se hacen los tests de estabilidad de los coeficientes de las variables 'dummy' estacionales trimestre por trimestre y en conjunto. Como puede observarse, en ninguno de todos los casos se rechaza la hipótesis nula de estacionariedad al nivel de $5 \%$ de significación. Esto indica que la serie es estacionaria tanto en la frecuencia cero cuanto en las frecuencias estacionales. Estos resultados son coherentes con aquellos obtenidos al usar el test de HEGY.

Comparando nuevamente con los resultados obtenidos en el caso de la aplicación de la metodologia de $\mathrm{CH}$ a la serie de precios de São Paulo, Aguirre y Sansó (1999) encuentran evidencias de existencia de una raíz unitaria estacional en la frecuencia $\pi$ de la serie brasileña. Tales resultados contradicen aquellos producidos - para la misma serie-por los tests de HEGY. Como em ambas metodologías la hipótesis nula es rechazada, Aguirre y Sansó eliminan la posibilidad de que la causa de esta incoherencia sea la baja potencia de los tests, concluyendo que debe tratarse de una especificación incorrecta del modelo utilizado para efectuarlos. El verdadero modelo podría ser, por ejemplo, no lineal.

\section{Conclusiones}

En este trabajo se analizan algunas propiedades estadísticas de la serie trimestral de precios del novillo en el mercado de Liniers. En particular, se usan dos metodologías alternativas para tratar de establecer si la serie tiene algún componente estacional significativo, si el mismo es determinístico o estocástico y, en este último caso, si es estacionario o intẻgrado.

Los resultados de los dos conjuntos de tests son coherentes e indican que la serie no tiene un patrón estacional determinístico significativo ni tampoco raíces unitarias estacionales. Por lo tanto, cualquier variación estacional existente en los datos es del tipo estocástica y estacionaria. Esta 
característica es diferente de la encontrada en la serie de precios del novillo en el Estado de São Paulo (Brasil). Esta última presenta estacionalidad determinística significativa, una raíz unitaria en la frecuencia cero y estacionalidad estocástica también estacionaria. Además, en el caso brasileño las conclusiones de los dos tipos de tests son incoherentes entre sí. Tal incoherencia puede deberse a la insuficiencia de datos para discriminar entre las dos hipótesis o a la especificación incorrecta del modelo usado para llevar a cabo el test (Aguirre y Sansó, 1999).

\section{APENDICE I}

En el caso de datos trimestrales, el operador de diferencia estacional $\left(1-L^{4}\right)$ puede ser factoreado usando sus raíces, como sigue:

$$
\left(1-L^{4}\right)=(1-L)(1+L)(1-i L)(1+i L)
$$

Cada una de las cuatro posibles raíces del proceso $(+1,-1, i,-i)$ corresponde a un ciclo en el dominio del tiempo. El periodo del ciclo asociado a cada raíz puede ser determinado aplicando el factor correspondiente de la raíz en cuestión a la serie $y_{t}$, por ejemplo, y observando el número de periodos necesarios para completar un ciclo.

La raíz + 1 tiene un ciclo que se completa en un periodo ("a singleperiod cycle'). Esto corresponde, en el dominio de la frecuencia, a la frecuencia cero y, por lo tanto, describe el comportamiento de largo plazo de la serie.

La raíz - 1 tiene un ciclo de dos trimestres, o sea, existen dos ciclos completos por año. Con efecto, la raíz-1 está asociada con el factor $(1+L)$ (ver ecuación 7.2 del texto) lo cual permite escribir:

$$
\begin{gathered}
(1+L) y_{t}=y_{1}+y_{t-1}=0 \\
y_{1}=-y_{t-1}
\end{gathered}
$$

Escribiendo la igualdad correspondiente al período siguiente, tenemos: 


$$
\begin{aligned}
y_{1+1} & =-y_{t} \\
& =-\left(-y_{t-1}\right) \\
& =y_{t-1}
\end{aligned}
$$

Adelantando el índice del tiempo más un periodo, tenemos:

$$
y_{1+2}=y_{1}
$$

De esta forma vemos que en dos períodos (en nuestro caso dos trimestres) se completa un ciclo (se alcanza el valor original $y_{1}$ ). Por ese motivo se dice que la raíz _ 1 está relacionada con un ciclo de período igual a seis meses, también denominado componente semianual.

Si un ciclo se completa en dos trimestres, al final del primer trimestre habrá transcurrido apenas la mitad del mencionado ciclo. Luego, como $\frac{1}{2} \times 2 \pi=\pi$, se acostumbra decir que la raíz -1 está asociada con la "frecuencia $\pi$ ".

El efecto de las dos raíces conjugadas es indistinguible y, por ese motivo, HEGY (1990) sugieren que ambas sean tratadas de manera conjunta en un ciclo anual.

Repitiendo el ejercicio anterior para el caso del polinomio:

$$
\begin{gathered}
\left(1+L^{2}\right) y_{t}=0 \\
\text { como } \frac{1}{4} \times 2 \pi=\frac{\pi}{2} \text { se acostumbra decir que las raíces conjugadas }
\end{gathered}
$$

complejas están asociadas con la "frecuencia $\frac{\pi}{2}$ ". Las raíces complejas conjugadas están asociadas con un ciclo de cuatro trimestres. 


\section{BIBLIOGRAFIA}

Aguirre, A., "Uma Introdução à Análise Espectral de Séries Temporais Econômicas", Nova Economia, Belo Horizonte, 5(1):41-60, 1995. , "Testing for seasonal unit roots in a quarterly series of beef cattle prices in the State of São Paulo (Brazil)", Revista de Economia e Sociologia Rural, 35(4):151-173, 1997.

Aguirre, A. y Sansó, A., Using different null hypotheses to test for seasonal unit roots in economic time series. CEDEPLAR/UFMG, Belo Horizonte, Texto para Discusión № 124, 1999.

Beaulieu, J. J. y Miron, J. A., "A Cross Country Comparison of Seasonal Cycles and Business Cycles", The Economic Journal, 102:772-788, 1992.

, "Seasonal Unit Roots in Aggregate U.S. Data", Journal of Econometrics, 55:305-328, 1993.

Canova, F. y Hansen, B. E., "Are Seasonal Patterns Constant over Time? A test for Seasonal Stability", Journal of Business and Economic Statistics, 13(3):237-252, 1995.

Chow, G. C., "Tests of equality between sets of coefficients in two linear regressions", Econométrica, 28:591-605, 1960.

Dickey, D. A. y Fuller, W. A., "Distributions of the estimators for autoregressive time series with a unit root", Journal of the American Statistical Association, 74:427-431, 1979.

-, Likelihood Ratio Statistics for Autoregressive Time Series with a Unit Root, Econométrica, 49(4):1057-1072, 1981.

Dickey, D. A. y Pantula, S. G., "Determining the Order of Differencing in Autoregressive Processes", Journal of Business and Economic Statistics, 5(4):455-461, 1987.

Engle, R. F., Granger, C. W. J. y Hallman, J., "Merging Short- and LongRun Forecasts", Journal of Econometrics, 40:45-62, 1989.

Ghysels, E. On the Economics and Econometrics of Seasonality, in: SIMS, C. (editor) Advances in Econometrics, Sixth World Congress of the Econometric Society, Cambridge University Press, Cambridge, UK, capítulo 7, pág. 257-316, 1994.

Haache, G., "The demand for money in the United Kingdom: evidence since 1971", Bank of England Quarterly Bulletin,14:284-305, 1974.

Harvey, A. y Scott, A., "Seasonality in Dynamic Regression Models", 
Economic Journal, 104 (427):1324-1345, 1994.

Hylleberg, S., (editor) Modelling Seasonality. Oxford University Press, Oxford, 1992.

Hylleberg, S., Engle, R. F., Granger, C. W. J. y Yoo, B. S., Seasonal Integration and Cointegration, Journal of Econometrics, 44(2):215-238, 1990.

Hylleberg, S., Jorgensen, C. y Sorensen, N. K., "Seasonality in economic time series", Empirical Economics, 18(2):321-335, 1993.

Ilmakunnas, P., "Testing the Order of Differencing in Quarterly Data: An Illustration of the Testing Sequence", Oxford Bulletin of Economics and Statistics, 52:79-88, 1990.

Miron, J. A., "The Economics of Seasonal Cycles", in: SIMS, C. (editor) Advances in Econometrics. Sixth World Congress. Cambridge University Press. Cambridge, UK, capítulo 6, pág. 213-251, 1994.

Nores, G., The economics of the cattle sector in Argentina, tesis de doctorado no publicada, Universidad de Purdue, EEUU, 1972.

Osborn, D. R., A survey of seasonality in UK macroeconomic variables, International Journal of Forecasting, 6:327-336, 1990.

Osborn, D. R., Chui, A. P. L., Smith, J. P y Birchenhall, C. R., Seasonality and the Order of Integration for Consumption. Oxford Bulletin of Economics and Statistics, 50:361-377, 1988.

Priestley, M. B., The Spectral Analysis of Time Series. Academic Press. London, 1981.

SAGyP-Secretaría de Agricultura, Ganadería y Pesca (1994) Estadísticas agropecuarias y pesqueras, Sistema integrado de información agropecuaria y pesquera, Buenos Aires. 